\title{
COMPARATIVE EFFECTS OF CLINIC- AND VIRTUAL REALITY-BASED MCKENZIE EXTENSION THERAPY IN CHRONIC NON-SPECIFIC LOW-BACK PAIN
}

original paper

( ) University School of Physical Education in Wroclaw

DOI: https://doi.org/10.5114/hm.2019.83998

CHIDOZIE EMMANUEL MBADA ${ }^{1}$, MOSES OLUWATOSIN MAKINDE ${ }^{1}$, ADESOLA CHRISTIANAH ODOLE ${ }^{2}$, OLUMIDE OLASUNKANMI DADA ${ }^{2}$, OLUSOLA AYANNIYI $^{2}$, AYOMIDE JOHNSON SALAMI ${ }^{1}$, ISHAYA PENI GAMBO ${ }^{3}$

${ }^{1}$ Department of Medical Rehabilitation, College of Health Sciences, Obafemi Awolowo University, Ile-Ife, Nigeria

${ }^{2}$ Department of Physiotherapy, College of Medicine, University of Ibadan, Ibadan, Nigeria

${ }^{3}$ Department of Computer Science and Engineering, Faculty of Technology, Obafemi Awolowo University, Ile-Ife, Nigeria

\begin{abstract}
Purpose. The study compared the influence of Clinic-Based McKenzie Therapy (CBMT) and a Virtual Reality Game (VRG) version on pain intensity, back extensor muscles endurance, activity limitation, participation restriction, fear avoidance belief, kinesiophobia, and general health status of patients with chronic non-specific low-back pain.

Methods. This quasi-experimental study involved 46 patients (CBMT: $n=24$; VRG: $n=22)$ with 'directional preference' for extension, randomized into CBMT or VRG group. Treatment was applied thrice weekly for 8 weeks. Outcomes were assessed at the end of the $4^{\text {th }}$ and $8^{\text {th }}$ week. Data analysis employed descriptive and inferential statistics of independent $t$-test, MannWhitney U test, repeated measure ANOVA, Friedman's ANOVA, and ANCOVA. The significance level was set as $\alpha=0.05$. Results. There were no significant differences in the treatment outcomes (mean change) across the groups ( $p>0.05)$, except for kinesiophobia, where VRG led to a significantly higher decline in mean rank at week $4(28.3$ vs. $19.1 ; p=0.018)$ and 8 (28.7 vs. 18.7; $p=0.009)$, and vitality (a general health status item) at week 4 (27.6 vs. 19.8; $p=0.042)$ and 8 (28.1 vs. 19.3 ; $p=0.042)$. ANCOVA showed that significant baseline parameters were not significant predictors of vitality $(\mathrm{F}=1.986 ; p=0.070)$ or kinesiophobia $(\mathrm{F}=0.866 ; \mathrm{p}=0.563)$ outcomes.
\end{abstract}

Conclusions. The VRG mode of McKenzie therapy is comparable with the clinic-based approach in most outcomes. VRG has a superior effect on kinesiophobia, but may take a higher toll on vitality/energy.

Key words: low-back pain, McKenzie therapy, virtual reality

\section{Introduction}

Low-back pain (LBP) is a major public health burden throughout the world [1], and it remains one of the most common problems in medical practice, affecting about $70-80 \%$ of adults during their lives [1]. LBP as a costly health problem concerns both the physiological and psychosocial aspects of the patient [2]; it is characterized by exacerbated nociceptive sensations, decreased physical performance, impaired psychosocial functioning, and work disability [3]. The Global Burden of Disease 2010 study estimated that LBP results in greater global disability than any other condition [4].
Specifically, on the basis of disability-adjusted life years, LBP ranked highest in terms of disability and $6^{\text {th }}$ in terms of overall burden out of 291 conditions studied [4]. It is adduced that the recent increase in population ageing is one of the major causes of the rise in global prevalence of LBP [5].

There have been praiseworthy advances in surgical, pharmacological, and physical management but for a limited number of patients. As such, LBP has remained disturbingly prevalent and has maintained a defiant stance against various therapeutic strategies [6]. Nonetheless, exercise therapy, which is the central element of physical therapy for LBP [7, 8], has been

Correspondence address: Chidozie Emmanuel Mbada, Department of Medical Rehabilitation, College of Health Sciences, Obafemi Awolowo University, PMB 13, Ile-Ife, Nigeria, e-mail: doziembada@yahoo.com

Received: June 12, 2018

Accepted for publication: February 25, 2019

Citation: Mbada CE, Makinde MO, Odole AC, Dada OO, Ayanniyi O, Salami AJ, Gambo IP. Comparative effects of clinicand virtual reality-based McKenzie extension therapy in chronic non-specific low-back pain. Hum Mov. 2019;20(3):66-79; doi: https://doi.org/10.5114/hm.2019.83998. 
recommended from systematic reviews as effective, especially in chronic or long-term LBP [8]. More so, exercise therapy is arguably the cheapest physiotherapeutic intervention and one in which the patient has some measure of direct control [9]. However, it remains inconclusive which exercise type is better than another [8], therefore leading to a proliferation of exercise practices with limited evidence on their effectiveness.

The McKenzie exercises are one of the most frequently used and effective types of physical therapy for patients with long-term LBP in some Western nations [10]. The Mechanical Diagnosis and Therapy (MDT), as a classification-based treatment, has the potential advantage of encouraging self-help [11]. However, proper evaluation and appropriate implementation of the McKenzie protocol are premised on specialized training in MDT [12, 13]. Czajka et al. [13] submit that 'a complete training regarding this method is significant for achieving high effectiveness of diagnosis. The combination of McKenzie Method with other forms of therapy gave the best results in improving spinal mobility and general quality of life as well as reducing the level of disability' (p. 5). Therefore, access to MDT can be limited owing to lack of skills in the techniques, geographical remoteness, and shortage of services in rural and distant areas [14]. Telerehabilitation is recommended as a solution for providing physiotherapy in settings with constrained access to rehabilitation services. Telerehabilitation is considered as a viable link that may help remedy the challenges of barriers of distance, time, and travel to receive care [15]. It may also help bridge the gap in the non-availability of faculties, especially for MDT. However, the validity of telerehabilitation for LBP compared with clinic-based approaches such as MDT is largely unproven.

Apart from the use of mobile phone-based applications in LBP, virtual reality involving video games or non-game applications to treat patients is an attractive alternative to conventional face-to-face physical therapy [16]. Studies on virtual reality as a low-cost, homebased tool to supplement traditional rehabilitation in LBP are just emerging. Virtual reality gaming in physical therapy offers a potential means of enhancing patient engagement in usual care or traditional therapy [16]. The efficacy of McKenzie therapy on fun mode using Virtual Reality Game (VRG) is yet to be explored. Therefore, the aim of this study was to compare the effectiveness of Clinic-Based McKenzie Therapy (CBMT) with virtual reality-based McKenzie therapy on pain intensity (PI), back extensor muscles endurance (BEME), activity limitation (AL), participation restriction (PR), fear avoidance belief (FAB), kinesiophobia (Kp), and general health status (GHS).

\section{Material and methods}

\section{Participants}

The participants in this study were patients with chronic non-specific LBP, consecutively recruited from selected physiotherapy facilities, namely: Obafemi Awolowo University (OAU) Teaching Hospital, Ile Ife, Nigeria (OAUTHC); Department of Medical Rehabilitation, OAU; and Ladoke Akintola University of Technology Teaching Hospital, Osogbo, Nigeria (LAUTECH). The sample size for the study was calculated by using the formula by Chan [17]:

$$
n=C \times \frac{\pi 1(1-\pi 1)+\pi 2(1-\pi 2)}{(\pi 1-\pi 2)^{2}}
$$

where $C=7.9$ for $80 \%$ power, and $\pi 1$ and $\pi 2$ are proportion estimates $(\pi 1=0.25$ and $\pi 2=0.65)$.

Thus: $n=7.9 \times \frac{0.25(1-0.25)+0.65(1-0.65)}{(0.25-0.65)^{2}}=20.49 \approx 21$ per group. In order to account for possible attrition, $10 \%$ of the calculated sample size was added, yielding a total of 46 (23 per group).

Eligible participants for this study were patients with long-term mechanical LBP. Long term mechanical LBP refers to back pain lasting not less than 3 months, of musculoskeletal origin, and with symptoms varying with physical activity [18]. The standard McKenzie Institute Lumbar Spine Assessment Algorithm (MILSAA) was used to determine eligibility criteria to participate in the study. On the basis of MILSAA, patients who demonstrated 'directional preference' (DP) for extension only were recruited in order to ensure homogeneity of samples. DP is described as the posture or movement that reduces or centralizes radiating pain which emanates from the spine. Patients with DP for flexion, lateral or no DP were excluded. Previous reports indicate that DP is substantially important in the derangement group of patients with mechanical LBP and they may present with a good prognosis with extension exercise [19, 20].

In addition, excluded were patients with positive history of red flags indicative of serious spinal pathology (i.e. those with complaints of dermatomal sensory loss, myotomal muscle weakness, and reduced lower limb reflexes); any obvious spinal deformity or neurological disease; pregnancy; previous spinal surgery; previous experience of MDT extension protocol; underlying systemic or visceral disease or specific condition such as dementia, cognitive dysfunction, visual impairment, history of epilepsy. 


\section{HUMAN MOVEMENT}

C. Mbada et al., Virtual reality-based rehabilitation

\section{Design}

The study utilized a randomized controlled trial design. It involved an experimental and control arm. The experimental group received the Back Extension-VRG (BE-VRG). The control group received CBMT. In order to ensure equal-sized treatment groups, random permuted blocks were used [21], with a block size of 4 chosen for the study. Computer-generated block permutations for 4 blocks of 2 groups (i.e. 4 factorial - (4!) / ((2!)(2!)) yielded 24 permutations (such as AABB, ABAB, and all other possible restricted permutations). The process of drawing block permuted sequence and randomization was repeated as participants were recruited. Single blind approach where only the researchers knew whether a patient was receiving conventional treatment or the new one, served to reduce bias. The study was designed to minimize both Hawthorne (Hawthorne effect implies a situation where participants show uncommonly higher levels of performance just because they know they are being studied) and spillover (spillover bias refers to an indirect effect occurring in those to whom intervention was not intended getting to know of interventions being investigated) effects by geographically separating the control from the intervention group. The participants in the control arm of the study were recruited and treated at the Physiotherapy Departments of LAUTECH and OAUTHC, while those in the intervention arms were treated at the Gymnasium of the Department of Medical Rehabilitation. Figure 1 shows the Consort diagram of recruitment and randomization of patients in the study.

\section{Pre-treatment screening}

In accordance with the bio-psychosocial framework and recommendations of review panels on LBP management, the study investigated the effects of the interventions by using standardized outcome measures on pain and other impairments, functional status, and general health measures [22].

Baseline assessment was carried out for each participant recruited to the study. Anthropometric variables like weight and height were measured. The subjects were screened for their eligibility to participate in the study: the inclusion and exclusion criteria as earlier specified were applied. Information such as age, gender, educational level, occupation, marital status, onset of back pain, recurrence, duration of complaint, previous intervention were recorded for each participant accordingly.
Following their qualification to participate in the study, each participant was requested to complete the outcome measures before the commencement of the exercise protocol and subsequently at week 4 and 8 of the study.

\section{Outcome measures}

The following outcome tools were used in the study:

\section{Quadruple Visual Analogue Scale (QVAS)}

QVAS is a variant of the visual analogue scale (VAS) but assesses pain experienced by the participants at the time of assessment, usual or average pain, pain at its best (least pain), and pain at its worst (maximum or peak pain) [23]. The tool is adjudged to mitigate recall bias and memory artifact associated with chronic pain, and thus is considered an improvement over VAS [23].

\section{Oswestry Disability Index (ODI)}

ODI is one of the most commonly used disability scales [24]. The tool covers 10 domains, including PI, personal care, lifting, walking, sitting, standing, sleeping, sex life, social life, and traveling. There is evidence that ODI is a valid and acceptable measurement tool for detecting disability related to LBP and that it is responsive to change [24, 25]. This tool was used to assess PR.

\section{Roland Morris Low Back Pain Disability Questionnaire (RMLBDQ)}

RMLBDQ is a 24-item questionnaire developed to measure self-rated disability due to back pain. The tool is reported to have adequate psychometric properties and is suitable for use in clinical settings to evaluate change in physical functioning in subjects with LBP [26, 27]. It was used to assess the participants' AL.

\section{The SF-12 General Health Status Questionnaire}

This tool is a 12-item questionnaire developed from the SF-36 Health Survey. Both the SF-36 and SF-12 are generic questionnaires, not designed for any special patient category, but recommended in the studies of back pain $[28,29]$. The scales of the tools include physical functioning, role limitations due to physical problems, bodily pain, general health perceptions, vitality, social functioning, role limitation due to emotional problems, and general mental health. On the trans- 
formed scores of the tools, lower scores represent poorer health and higher scores represent better health. The primary advantage of the SF-12 over the 36-item version is its brevity and improved practicality [30]. The SF-12 was used to assess the participants' GHS.

\section{Fear Avoidance Beliefs Questionnaire (FABQ)}

FABQ was developed to measure pain-related fear of physical activity that causes avoidance of activity and increased disability [31]. The 7-item scale has satisfactory psychometric properties. The higher the scale scores, the greater the degree of fear and avoidance beliefs shown by the patient. The tool describes the fear of movement in patients at work and during physical activity. FABQ was used to assess the participants' fear of movement.

\section{Tampa Scale for Kinesiophobia (TSK)}

This is a 17-item scale used to assess fear of movement. It is a self-administered questionnaire and its scores range from 17 to 68 , with higher scores indicating an increasing degree of $\mathrm{Kp}$. This scale was developed to assess fear of movement associated with pain related to different parts of the body [32].

\section{Physical performance test}

The physical performance assessment for static BEME was conducted prior to the commencement of treatment intervention and at the $4^{\text {th }}$ and $8^{\text {th }}$ week of treatment. The evaluation employed the modified Biering-Sørensen test of Static Muscular Endurance (BSME). Prior to the endurance test, the participants were instructed in detail on the study procedures. The test was to be preceded by a low-intensity warm-up phase of 5 minutes that comprised stretches and strolling at a self-determined pace around the research venue. The test was also to be ended with a cool-down phase, comprising the same low-intensity stretches and strolling around the research venue for about 5 minutes.

\section{Assessment of static back extensor muscles} endurance

BSME was used to assess the static BEME. During the test, the participant lay on a plinth in the prone position, with the upper edge of the iliac crests aligned with the edge of the plinth, and hands held by the sides. The lower body was fixed to the plinth by two non-elastic straps around the pelvis and ankles. Horizontality in the test position was ensured by asking the participant to maintain contact between their back and a hanging weighted ball. Once a loss of contact for more than 10 seconds was noticed, the participant was encouraged to immediately maintain the contact again. Once the participant could not immediately correct or hold the position or claimed to be fatigued, the test was terminated [33, 34].

\section{Procedure}

Two interventions, namely the CBMT and the BEVRG, were tested to determine their effectiveness in this study. Each intervention comprised a warm-up, main intervention, and cool-down phase. The warmup and the cool-down involved low-intensity active stretching of the upper extremities and low back for about 5 minutes. The active stretch was carried out in a top-to-bottom sequence, involving overhead triceps and arm-cross-chest stretch, forearm flexion and extension contraction, and torso twist stretch performed in routine in a balanced standing position. Both interventions were carried out thrice weekly for 8-week treatment. The outcomes were assessed at the end of the $4^{\text {th }}$ and $8^{\text {th }}$ week.

\section{Clinic-Based McKenzie Therapy}

The participants in the CBMT group received the McKenzie extension in standing protocol. The protocol involves a course of specific lumbosacral repeated movements in extension that cause the symptoms to centralize, decrease, or abolish [35]. The McKenzie extension protocol has different starting positions; however, in order to ensure comparability in orientation among interventions, the CBMT in this study was delimited to the standing position.

During the extension in standing, the participants were asked to stand upright with the feet slightly apart and to place their hands in the small of the back with the fingers pointing backwards. The participant then stretched the trunk backwards at the waist level as far as they could, using the hands as a fulcrum while keeping the knees straight. The movement was repeated up to 10 times. In addition to the MDT extension protocol, the participants in the CBMT group received a set of back care education instructions comprising a 9-item instructional guide on standing, sitting, lifting, and other activities of daily living for home. 


\section{HUMAN MOVEMENT}

C. Mbada et al., Virtual reality-based rehabilitation

\section{Back Extension-Virtual Reality Game}

The BE-VRG is an interactive video game developed to be operational on the Microsoft Kinect Xbox, which allows for a less invasive 3-dimensional graphic environment on a computer screen, flat screen monitor, or projector screen. BE-VRG has virtual reality tasks developed and conducted on a Microsoft's Kinect platform. The choice of tasks in the BE-VRG was based on the intention that they had to be comparable with the McKenzie extension protocol, as well as relevant in rehabilitation of LBP. Thus, a VRG involving heading of a projectile virtual soccer ball was developed. Each participant was asked to maintain an upright standing position with the feet, while holding the waist with both hands. Stretching the trunk, the subject was asked to head balls coming to them as seen on the screen. The intended therapeutic motion was extension of the spine while standing. However, side-glides of the spine to the left and also to the right were observed as accompanying motions in that position. The participants were instructed to head away virtual balls coming towards them while their feet were stationary but the trunk and head moved to accomplish the ball heading. The trajectory of the balls was set in such a way that it elicited the required therapeutic movements (Figure 2). This BE-VRG was designed to provide a progression of increasingly difficult challenges that can help keep the players engaged and motivated over an extended period of time. During the game play, visual and textual feedback on the patient's performance and results was displayed on the screen. In addition to BE-VRG, the participant received the same back care education instructions as the control group. The result of the feasibility testing of the VRG in terms of usability, satisfaction, and treatment experience is being published elsewhere. Figure 2 shows a participant receiving BE-VRG.

\section{Data analysis}

Descriptive statistics of mean and standard deviation was used to summarize the data. An independent $t$-test was applied to compare demographic and continuous variables (QVAS, RMLBDQ, and BSME scores), while the Mann-Whitney $U$ test served to compare categorical variables (ODI, FABQ, TSK, and SF12 scores) between the two interventions. The chi-square test was used to compare the proportion of participants by gender. Repeated measure ANOVA was employed for within-group comparison of the effects of the different treatment regimens across baseline, $4^{\text {th }}$ and $8^{\text {th }}$ week for the continuous variables. Friedman's ANOVA was used for within-group comparison of the effects of the different treatment regimens across baseline, $4^{\text {th }}$ and $8^{\text {th }}$ week for the categorical variables. Analysis of covariance (ANCOVA) allowed to check the effects of co-founders on the outcomes of the study. The alpha level was set at $p<0.05$. The data analyses were carried out with the SPSS 20.0 version software (SPSS Inc., Chicago, USA).

\section{Randomization}

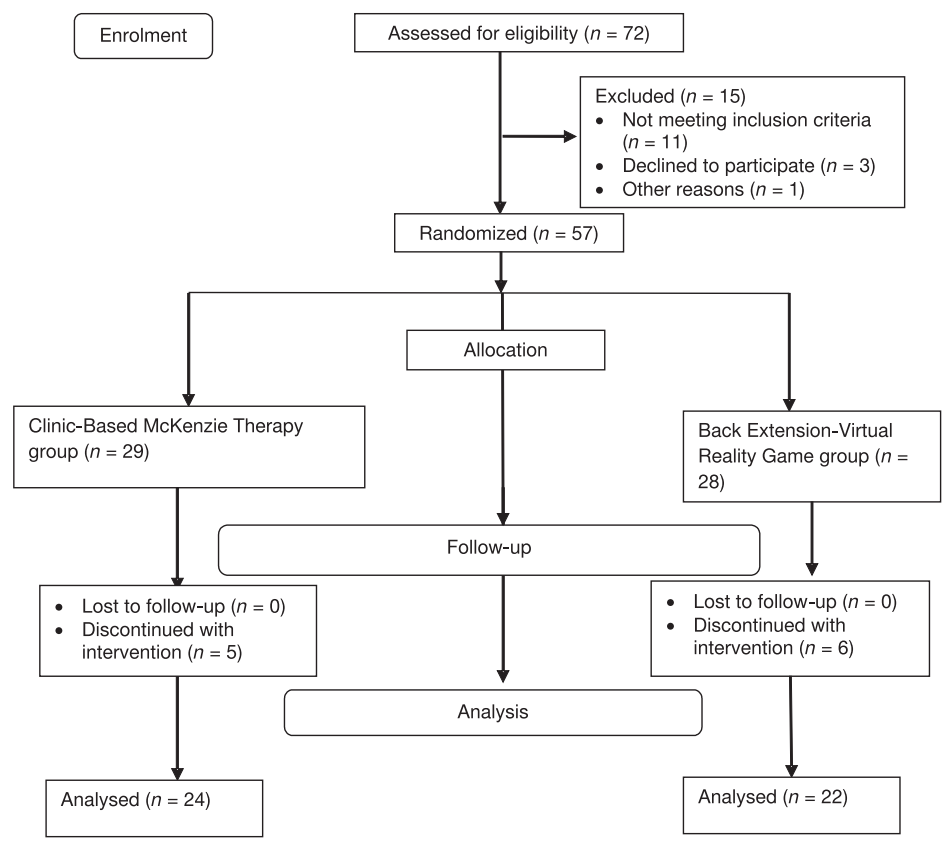

Figure 1. Consort diagram of the flow of patients through the study

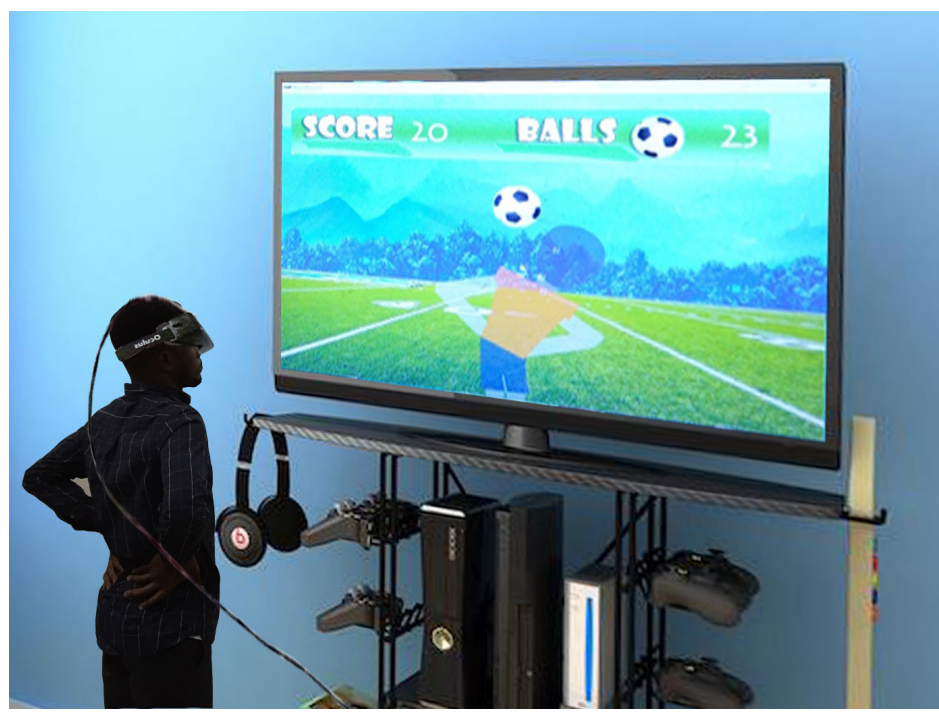

Figure 2. A participant in the Back Extension-Virtual Reality Game group 


\section{Ethical approval}

The research related to human use has complied with all the relevant national regulations and institutional policies, has followed the tenets of the Declaration of Helsinki, and has been approved by the Health Research Ethics Committee of the Institute of Public Health, Obafemi Awolowo University, Ile-Ife, Nigeria (IPHOAU/12/885).

\section{Informed consent}

Informed consent has been obtained from all individuals included in this study.

\section{Results}

The mean age and duration of participants' LBP was $41.1 \pm 13.5$ years and $8.2 \pm 3.7$ months, respectively. The two groups were comparable in their general characteristics $(p>0.05)$ except for age, gender, and height $(p<0.05)$ (Table 1). Comparison of the baseline measures across the groups is presented in Table 2. The participants in both groups were comparable in the variables $(p>0.05)$ except for static BEME $(p=0.023)$, PR $(p=0.025)$, fear of movement ( $p=0.001$ ), and some of the scales (physical function, role limitation-physical, bodily pain, and vitality/energy) of the SF-12 $(p<0.05)$.

Between-group comparison of CBMT and BE-VRG at week 4 of the study showed that there were no significant differences $(p>0.05)$ in the treatment outcome (mean change) (Table 3), except for fear of movement (28.3 vs. $19.1 ; p=0.018$ ) and the energy/fatigue scale on the SF-12 (27.6 vs. $19.8 ; p=0.042$ ), with BE-VRG having a significantly higher mean rank. However, CBMT presented a higher mean change in role limi- tation-physical (27.5 vs. $19.1 ; p=0.017$ ) (Table 3). Similarly, there were no significant differences $(p>0.05)$ in the treatment outcomes across both groups at the end of week 8 (i.e. change between baseline and week 8 outcomes) except for a higher mean change observed in fear of movement (28.7 vs. $18.7 ; p=0.009)$ and the energy/fatigue scale (28.1 vs. $19.3 ; p=0.042)$ in BEVRG (Table 4).

Within-group comparisons in the outcome parameters across the 3 time points (baseline, $4^{\text {th }}$ and $8^{\text {th }}$ week) of the study are presented in Tables 5 and 6 . There were significant differences $(p<0.05)$ in the outcome parameters of participants in the CBMT group, except for FAB (work) $(p=0.265)$. However, BE-VRG led to significant differences in all the outcome parameters $(p<0.05)$.

Analysis of covariance was carried out to determine whether the parameters that were significantly different at baseline between the groups constituted significant predictors of fear of movement and vitality/energy outcomes at the end of the study. The result indicates that age ( $p=0.889)$, gender $(p=0.059)$, height $(p=0.635)$, ODI score $(p=0.929), \operatorname{Kp}$ score $(p=$ $0.682)$, BSME level ( $p=0.891)$, SF-12 physical function score $(p=0.585)$, SF-12 role limitation-physical score $(p=0.064)$, and SF-12 bodily pain score $(p=$ $0.572)$ were not significant $(\mathrm{F}=1.986 ; p=0.070)$ predictors of vitality/energy at week 8 . Similarly, the study showed that that age $(p=0.299)$, gender $(p=$ $0.327)$, height $(p=0.159)$, ODI score $(p=0.922)$, BSME level ( $p=0.431)$, SF-12 physical function score $(p=$ $0.394)$, SF-12 role limitation-physical score $(p=0.693)$, SF-12 bodily pain score $(p=0.484)$ and SF-12 vitality/ fatigue score $(p=0.530)$ were not significant $(\mathrm{F}=0.866$; $p=0.563)$ predictors of $\mathrm{Kp}$ at week 8 .

Table 1. Comparison of the participants' general characteristics by treatment groups $(n=46)$

\begin{tabular}{|c|c|c|c|c|c|}
\hline Variable & $\begin{array}{l}\text { CBMT } \\
(n=24)\end{array}$ & $\begin{array}{l}\text { BE-VRG } \\
(n=22)\end{array}$ & Statistics & $p$ value & $\begin{array}{l}\text { All participants } \\
\qquad(n=46)\end{array}$ \\
\hline & $\bar{x} \pm S D$ & $\bar{x} \pm S D$ & $t$ & & $\bar{x} \pm S D$ \\
\hline Age (years) & $48.8 \pm 10.2$ & $32.6 \pm 11.5$ & 5.070 & $0.001^{*}$ & $41.1 \pm 13.5$ \\
\hline Height (m) & $1.60 \pm 0.10$ & $1.70 \pm 0.10$ & -2.396 & $0.021^{*}$ & $1.70 \pm 0.10$ \\
\hline Weight (kg) & $70.9 \pm 7.90$ & $72.9 \pm 14.0$ & -0.615 & 0.542 & $71.9 \pm 11.2$ \\
\hline $\operatorname{BMI}\left(\mathrm{kg} / \mathrm{m}^{2}\right)$ & $26.4 \pm 3.60$ & $25.3 \pm 4.80$ & 0.953 & 0.346 & $25.9 \pm 4.20$ \\
\hline Pain duration (months) & $8.40 \pm 3.30$ & $8.00 \pm 4.20$ & 0.374 & 0.710 & $8.20 \pm 3.70$ \\
\hline Gender & $n(\%)$ & $n(\%)$ & $\chi^{2}$ & & $n(\%)$ \\
\hline Male & $5(20.8)$ & $12(54.5)$ & 5.599 & $0.018 *$ & $17(37.0)$ \\
\hline Female & $19(79.2)$ & $10(45.5)$ & & & $29(63.0)$ \\
\hline
\end{tabular}

* significant difference, $p<0.05$

CBMT - Clinic-Based McKenzie Therapy, BE-VRG - Back Extension-Virtual Reality Game, $\bar{x}-$ mean,

$S D$ - standard deviation, BMI - body mass index 


\section{HUMAN MOVEMENT}

C. Mbada et al., Virtual reality-based rehabilitation

Table 2. Comparison of the participants' baseline pain, activity limitation, back extensors endurance, participation restriction, fear avoidance belief, fear of movement, and general health status

\begin{tabular}{|c|c|c|c|c|}
\hline Variable & $\mathrm{CBMT}(n=24)$ & $\operatorname{BE}-\operatorname{VRG}(n=22)$ & Statistics & $p$ value \\
\hline Parametric & $\bar{x} \pm S D$ & $\bar{x} \pm S D$ & $t$ value & \\
\hline \multicolumn{5}{|l|}{ Pain intensity } \\
\hline Right now & $5.0 \pm 1.9$ & $4.1 \pm 1.8$ & 1.674 & 0.870 \\
\hline Average & $5.3 \pm 1.5$ & $4.8 \pm 1.2$ & 1.323 & 0.147 \\
\hline At its best & $2.6 \pm 1.3$ & $2.6 \pm 1.3$ & -0.030 & 0.744 \\
\hline At its worst & $7.1 \pm 1.4$ & $6.9 \pm 1.4$ & 0.311 & 0.624 \\
\hline QVAS score & $58.1 \pm 14.3$ & $52.9 \pm 11.4$ & 1.346 & 0.171 \\
\hline Activity limitation & $11.3 \pm 4.60$ & $6.8 \pm 4.9$ & 3.185 & 0.853 \\
\hline BSME & $20.6 \pm 13.3$ & $35.3 \pm 22.5$ & -2.722 & $0.023 *$ \\
\hline \multicolumn{5}{|l|}{ General health status } \\
\hline \multicolumn{5}{|l|}{ Scale } \\
\hline Physical function & $27.0 \pm 22.9$ & $45.5 \pm 25.3$ & -2.58 & $0.013 *$ \\
\hline Role limitation-physical & $97.1 \pm 9.9$ & $82.5 \pm 17.9$ & 3.458 & $0.001^{*}$ \\
\hline Bodily pain & $58.1 \pm 24.4$ & $72.9 \pm 15.5$ & -2.44 & $0.020 *$ \\
\hline Health perception & $40.2 \pm 26.6$ & $52.5 \pm 23.9$ & -1.64 & 0.107 \\
\hline Vitality/energy & $42.5 \pm 23.8$ & $60.0 \pm 21.4$ & -2.61 & $0.012 *$ \\
\hline Social functioning & $90.6 \pm 12.4$ & $87.5 \pm 12.8$ & 0.842 & 0.404 \\
\hline Role limitation-emotional & $94.2 \pm 13.3$ & $87.3 \pm 17.2$ & 1.530 & 0.134 \\
\hline Mental health & $52.8 \pm 13.9$ & $52.5 \pm 13.7$ & 0.061 & 0.952 \\
\hline \multicolumn{5}{|l|}{ Domain } \\
\hline Mental health & $70.0 \pm 9.05$ & $71.8 \pm 5.97$ & 0.792 & 0.433 \\
\hline Physical health & $64.3 \pm 10.81$ & $68.8 \pm 6.12$ & -1.72 & 0.092 \\
\hline Non-parametric & MR & MR & $U$ & \\
\hline Participation restriction & 27.8 & 18.7 & 162.0 & $0.025^{*}$ \\
\hline \multicolumn{5}{|l|}{ Fear avoidance belief } \\
\hline At work & 25.7 & 21.2 & 212.5 & 0.227 \\
\hline In physical activities & 25.8 & 21.0 & 208.5 & 0.193 \\
\hline Fear of movement & 29.9 & 16.5 & 110.0 & $0.001 *$ \\
\hline
\end{tabular}

* significant difference, $p<0.05$

CBMT - Clinic-Based McKenzie Therapy, BE-VRG - Back Extension-Virtual Reality Game, $\bar{x}-$ mean, $S D$ - standard deviation, QVAS - Quadruple Visual Analogue Scale, BSME - Biering-Sørensen test of Static Muscular Endurance,

MR - mean ranked, $U$ - Mann-Whitney $U$ test

\section{Discussion}

The study compared the effect of CBMT and BEVRG on PI, BEME, AL, PR, FAB, Kp, and GHS among patients with chronic non-specific LBP who had DP for extension. The average age of the patients was 40 years, which is within the age bracket of $40-80$ years of LBP prevalence [36]. The within-group comparison of the patients in the CBMT and BE-VRG groups across the 3 time points of the study revealed that both interventions had significant effects on all the outcome parameters. Traditionally, the McKenzie protocol is a clinicbased intervention but with a self-help approach where the patients are required to lie prone initially in a neu- tral or near neutral position and then, as the pain subsides, they are brought passively into greater and greater amounts of lumbar extension. Consequently, there are plenitude of studies on the clinic-based application of the McKenzie protocol with evidence about its efficacy on pain [19,37,38], disability [39, 40], quality of life $[40,41]$, as well as other parameters, such as patient satisfaction [42].

In explaining the therapeutic effects of the McKenzie protocol, McKenzie [35] claims that in the derangement syndrome, spinal flexion causes a movement of the nucleus pulposus to a more posterior position owing to increased mechanical compression on the anterior surface of the intervertebral disc [35], 
Table 3. Comparison of the participants' treatment outcomes (mean change) at week 4 of the study

\begin{tabular}{|c|c|c|c|c|}
\hline Variable & CBMT $(n=24)$ & $\operatorname{BE}-\operatorname{VRG}(n=22)$ & Statistics & $p$ value \\
\hline Parametric & $\bar{x} \pm S D$ & $\bar{x} \pm S D$ & $t$ value & \\
\hline \multicolumn{5}{|l|}{ Pain intensity } \\
\hline Right now & $1.6 \pm 1.5$ & $1.9 \pm 1.5$ & -0.640 & 0.194 \\
\hline Average & $1.8 \pm 1.5$ & $1.8 \pm 1.5$ & 0.035 & 0.584 \\
\hline At its best & $1.4 \pm 1.0$ & $1.3 \pm 1.5$ & 0.386 & 0.221 \\
\hline At its worst & $2.5 \pm 1.6$ & $2.1 \pm 1.9$ & 0.628 & 0.858 \\
\hline QVAS score & $19.7 \pm 12.6$ & $19.6 \pm 13.7$ & 0.044 & 0.558 \\
\hline Activity limitation & $5.2 \pm 3.0$ & $3.1 \pm 4.5$ & 1.818 & 0.675 \\
\hline BSME & $-8.7 \pm 5.9$ & $-13.7 \pm 10.2$ & 2.100 & 0.120 \\
\hline \multicolumn{5}{|l|}{ General health status } \\
\hline \multicolumn{5}{|l|}{ Scale } \\
\hline Physical function & $33.3 \pm 28.2$ & $23.5 \pm 25.5$ & 1.24 & 0.223 \\
\hline Role limitation-physical & $20.4 \pm 17.6$ & $4.8 \pm 22.4$ & 2.64 & $0.011^{*}$ \\
\hline Bodily pain & $21.3 \pm 23.1$ & $8.4 \pm 18.4$ & 2.07 & $0.040^{*}$ \\
\hline Health perception & $30.6 \pm 38.7$ & $21.6 \pm 29.9$ & 0.88 & 0.384 \\
\hline Vitality/energy & $31.7 \pm 24.3$ & $17.3 \pm 25.7$ & 1.96 & 0.060 \\
\hline Social functioning & $11.5 \pm 12.7$ & $5.7 \pm 13.2$ & 1.51 & 0.138 \\
\hline Role limitation-emotional & $23.3 \pm 19.7$ & $14.3 \pm 20.7$ & 1.51 & 0.138 \\
\hline Mental health & $12.5 \pm 14.1$ & $7.2 \pm 16.5$ & 1.18 & 0.243 \\
\hline \multicolumn{5}{|l|}{ Domain } \\
\hline Mental health & $21.0 \pm 11.6$ & $14.4 \pm 12.3$ & 1.862 & 0.069 \\
\hline Physical health & $8.8 \pm 10.4$ & $7.61 \pm 8.69$ & 0.421 & 0.676 \\
\hline Non-parametric & MR & MR & $U$ & \\
\hline Participation restriction & 23.3 & 23.8 & 258.0 & 0.895 \\
\hline \multicolumn{5}{|l|}{ Fear avoidance belief } \\
\hline At work & 23.4 & 23.6 & 261.5 & 0.950 \\
\hline In physical activities & 22.7 & 24.4 & 244.5 & 0.648 \\
\hline Fear of movement & 19.1 & 28.3 & 158.0 & $0.018^{*}$ \\
\hline
\end{tabular}

* significant difference, $p<0.05$

CBMT - Clinic-Based McKenzie Therapy, BE-VRG - Back Extension-Virtual Reality Game, $\bar{x}-$ mean, $S D$ - standard deviation, QVAS - Quadruple Visual Analogue Scale, BSME - Biering-Sørensen test of Static Muscular Endurance, MR - mean ranked, $U$ - Mann-Whitney $U$ test

while extension alleviates stress on the posterior annulus, reduces nerve root compression, and, as a result, relieves pain [43]. Other authors [44] explain that nuclear pressure is reduced when compressive force is transferred from the vertebral disc body unit to the apophyseal joints during extension exercise. Furthermore, Adams et al. [37] posit that the posterior annulus can be stress-shielded by the neural arch in extended postures, and this may explain why extension exercises can relieve LBP in some patients.

In addition to the foregoing, some authors theorize that extension movements cause an anterior migration of nuclear tissue, which conversely displaces posteriorly during flexion [15]. Therefore, the therapeutic effect of the extension principle of the McKenzie method may be associated with the possibility of extension to cause internal displacements, as well as reduce posterior protrusions in some intervertebral discs [45]. Conversely to the previous explanations, extension movement is adduced to relieve pain by reducing the forces acting on pain-sensitive tissues [37]. Extension movement is hypothesized to unload the whole disc as the vertebrae can pivot around the apophyseal joints during the manoeuvre [37]. Also, within the disc itself, extension causes a transfer of load from the anterior annulus and nucleus to the posterior annulus and the effect is magnified after creep-loading. Sustained and repeated extension movements have been reported to increase the height of the spine presumably by unloading the disc and permitting rehydration [46]. 
Table 4. Comparison of the participants' treatment outcomes (mean change) at week 8 of the study

\begin{tabular}{|c|c|c|c|c|}
\hline Variable & $\operatorname{CBMT}(n=24)$ & BE-VRG $(n=22)$ & Statistics & $p$ value \\
\hline Parametric & $\bar{x} \pm S D$ & $\bar{x} \pm S D$ & $t$ value & \\
\hline \multicolumn{5}{|l|}{ Pain intensity } \\
\hline Right now & $3.5 \pm 2.3$ & $3.5 \pm 1.7$ & 0.001 & 0.056 \\
\hline Average & $3.6 \pm 1.6$ & $3.9 \pm 1.3$ & -0.662 & 0.140 \\
\hline At its best & $2.3 \pm 1.3$ & $2.4 \pm 1.2$ & -0.434 & 0.413 \\
\hline At its worst & $4.1 \pm 1.7$ & $4.9 \pm 1.9$ & -1.480 & 0.402 \\
\hline QVAS score & $37.2 \pm 16.4$ & $40.8 \pm 13.2$ & -0.804 & 0.099 \\
\hline Activity limitation & $8.9 \pm 4.1$ & $5.7 \pm 4.7$ & 2.422 & 0.871 \\
\hline BSME & $-15.1 \pm 7.9$ & $-21.9 \pm 14.6$ & 1.984 & 0.177 \\
\hline \multicolumn{5}{|l|}{ General health status } \\
\hline \multicolumn{5}{|l|}{ Scale } \\
\hline Physical function & $44.4 \pm 26.3$ & $31.8 \pm 30.0$ & 10.522 & 0.135 \\
\hline Role limitation-physical & $8.70 \pm 15.5$ & $3.18 \pm 18.4$ & 10.113 & 0.272 \\
\hline Bodily pain & $28.8 \pm 27.5$ & $19.8 \pm 16.5$ & 10.326 & 0.192 \\
\hline Health perception & $42.3 \pm 33.5$ & $33.4 \pm 23.8$ & 10.029 & 0.309 \\
\hline Vitality/energy & $45.8 \pm 32.6$ & $26.4 \pm 25.7$ & 20.236 & $0.030 *$ \\
\hline Social functioning & $13.5 \pm 12.7$ & $12.5 \pm 12.8$ & 00.277 & 0.783 \\
\hline Role limitation-emotional & $24.8 \pm 16.3$ & $19.1 \pm 17.8$ & 10.134 & 0.263 \\
\hline Mental health & $17.8 \pm 15.1$ & $11.7 \pm 16.5$ & 10.302 & 0.200 \\
\hline \multicolumn{5}{|l|}{ Domain } \\
\hline Mental health & $26.6 \pm 13.3$ & $19.4 \pm 11.6$ & 10.951 & 0.057 \\
\hline Physical health & $16.3 \pm 13.0$ & $11.6 \pm 8.70$ & 10.456 & 0.153 \\
\hline Non-parametric & MR & MR & $U$ & \\
\hline Participation restriction & 25.7 & 21.1 & 212.0 & 0.252 \\
\hline \multicolumn{5}{|l|}{ Fear avoidance belief } \\
\hline At work & 22.6 & 24.5 & 241.5 & 0.598 \\
\hline In physical activities & 2444 & 22.5 & 241.5 & 0.598 \\
\hline Fear of movement & 18.7 & 28.8 & 148.0 & $0.009 *$ \\
\hline
\end{tabular}

* significant difference, $p<0.05$

CBMT - Clinic-Based McKenzie Therapy, BE-VRG - Back Extension-Virtual Reality Game, $\bar{x}$ - mean, $S D$ - standard deviation, QVAS - Quadruple Visual Analogue Scale, BSME - Biering-Sørensen test of Static Muscular Endurance, MR - mean ranked, $U$ - Mann-Whitney $U$ test

The McKenzie method propagates the principle of extension in the management of LBP. Although its efficacy has been established by several studies, it is yet to be proven whether the method will produce comparable effect when administered on different platforms other than the traditional clinic-based approach. A study by Mbada et al. [47] indicates that a mobilephone application of the McKenzie extension protocol was comparable in effect with clinic-based methods in terms of PI, AL, PR, and GHS. However, there is an apparent dearth of studies on VRG application of the McKenzie protocol with which the findings of this study could be compared.

The BE-VRG used in this study had significant effects on PI, BEME, AL, PR, FAB, Kp, and GHS. The
BE-VRG was developed to be comparable in characteristics with CBMT. Specifically, VRG has some back extension-glide mix movement components that are embedded in a soccer game setting where the patient is expected to head virtual soccer balls so as to win points. The trajectory of the balls was set in such a way that it elicited the required therapeutic extension, however, with some slight right and left side glide movements. The repetition of movements involved in the BE-VRG is between 90 and 120 extension-glide, which is by far more in frequency than the CBMT movement of 10 repetitions. Movement training using visual feedback as obtained in VRGs has been shown to be effective in addressing pain and improving functional capacity in patients with chronic LBP [48]. For example, 
Table 5. Comparison of treatment outcomes in the CBMT group across the 3 time points of the study $(n=24)$

\begin{tabular}{|c|c|c|c|c|c|}
\hline Variable & Baseline & $4^{\text {th }}$ week & $8^{\text {th }}$ week & $\begin{array}{l}\text { Statistic } \\
\text { F-ratio } †\end{array}$ & $p$ value \\
\hline Parametric & $\bar{x} \pm S D$ & $\bar{x} \pm S D$ & $\bar{x} \pm S D$ & $t$ value & \\
\hline \multicolumn{6}{|l|}{ Pain intensity } \\
\hline Right now & $5.0 \pm 1.9$ & $3.4 \pm 0.8$ & $1.5 \pm 1.3$ & 253.2 & $0.001^{*}$ \\
\hline Average & $5.3 \pm 1.5$ & $3.5 \pm 1.1$ & $1.8 \pm 0.9$ & 364.1 & $0.001^{*}$ \\
\hline At its best & $2.6 \pm 1.3$ & $1.2 \pm 1.1$ & $0.8 \pm 0.7$ & 65.2 & $0.001^{*}$ \\
\hline At its worst & $7.1 \pm 1.4$ & $4.6 \pm 1.2$ & $3.0 \pm 1.1$ & 667.3 & $0.001^{*}$ \\
\hline QVAS score & $58.1 \pm 14.3$ & $38.3 \pm 8.4$ & $20.8 \pm 10.0$ & 523.8 & $0.001^{*}$ \\
\hline Activity limitation & $11.3 \pm 4.6$ & $6.3 \pm 2.9$ & $2.4 \pm 1.8$ & 145.6 & $0.001^{*}$ \\
\hline \multicolumn{6}{|l|}{ General health status } \\
\hline \multicolumn{6}{|l|}{ Scale } \\
\hline Physical function & $27.1 \pm 23.0$ & $60.4 \pm 20.7$ & $71.5 \pm 17.4$ & 68.5 & $0.001^{*}$ \\
\hline Role limitation-physical & $97.1 \pm 9.9$ & $76.7 \pm 16.9$ & $88.3 \pm 16.9$ & 7.67 & $0.011^{*}$ \\
\hline Bodily pain & $58.1 \pm 24.4$ & $79.4 \pm 13.7$ & $86.9 \pm 14.4$ & 26.2 & $0.001^{*}$ \\
\hline Health perception & $40.2 \pm 26.6$ & $70.8 \pm 26.9$ & $82.5 \pm 14.7$ & 38.3 & $0.001 *$ \\
\hline Vitality/energy & $42.5 \pm 23.8$ & $74.2 \pm 18.2$ & $88.3 \pm 20.4$ & 47.6 & $0.001^{*}$ \\
\hline Social functioning & $90.6 \pm 12.4$ & $79.2 \pm 9.5$ & $77.1 \pm 7.1$ & 27.2 & $0.001 *$ \\
\hline Role limitation-emotional & $94.2 \pm 13.3$ & $70.8 \pm 13.2$ & $69.4 \pm 11.8$ & 55.9 & $0.001^{*}$ \\
\hline Mental health & $52.8 \pm 13.9$ & $65.3 \pm 6.2$ & $70.6 \pm 4.4$ & 33.5 & $0.001^{*}$ \\
\hline \multicolumn{6}{|l|}{ Domain } \\
\hline Mental health & $70.0 \pm 9.05$ & $72.4 \pm 7.47$ & $76.3 \pm 6.47$ & 11.204 & $0.003^{*}$ \\
\hline Physical health & $64.3 \pm 10.8$ & $73.1 \pm 7.3$ & $80.6 \pm 7.1$ & 34.8 & $0.001^{*}$ \\
\hline Non-parametric & MR & MR & MR & $\chi^{2} \neq$ & \\
\hline Participation restriction & 2.9 & 2.0 & 1.0 & 44.1 & $0.001^{*}$ \\
\hline \multicolumn{6}{|l|}{ Fear avoidance belief } \\
\hline At work & 2.1 & 1.8 & 2.2 & 2.7 & 0.265 \\
\hline In physical activities & 1.5 & 1.6 & 2.9 & 31.5 & $0.001^{*}$ \\
\hline Fear of movement & 1.8 & 1.7 & 2.5 & 10.9 & $0.004^{*}$ \\
\hline
\end{tabular}

* significant difference, $p<0.05$, † repeated measures ANOVA, $\ddagger$ Friedman’s ANOVA

CBMT - Clinic-Based McKenzie Therapy, $\bar{x}$ - mean, SD - standard deviation, QVAS - Quadruple Visual Analogue Scale, $\mathrm{MR}$ - mean ranked

a study by Thomas et al. [49] validated virtual reality dodgeball on pain-related fear avoidance and trunk range of movement.

While studies on VRG in chronic LBP are just emerging, available research postulates that the effectiveness of the approach can be attributed to neuromodulatory mechanisms [50]. In explaining the neuromodulatory mechanisms, VRG interventions in chronic LBP are cognitive-behavioural approaches using graded exposure that could encourage movement of the lumbar spine, especially in highly avoidant and fearful patients with chronic LBP [51]. In a fun, motivating, and distracting context, BE-VRG exerts a neuromodulatory effect by stimulating patients to head virtual soccer balls while standing leg wide apart in order to head off the balls and, in the process, causing a sig- nificant amount of spinal extension and side glides. The fun component of the intervention influences the patients' cognition, as impairment of cognition is among the most common predictors of chronic pain and disability for patients with LBP [52]. Increases in movement elicited by VRGs may also be associated with the way movement is mapped in the brain in chronic pain [53]. For example, literature suggests that brain areas that code for movement in chronic LBP are dysfunctional, as there is impaired motor imagery performance with respect to the back [54]. It is adduced that visual feedback of movement obtainable in VRGs may play an important role in perception and could help re-calibrate the potential incongruence between actual movement and perceived movement in chronic pain conditions [55]. However, evidence for the incon- 
Table 6. Comparison of treatment outcomes in the BE-VRG group across the 3 time points of the study $(n=22)$

\begin{tabular}{|c|c|c|c|c|c|}
\hline Variable & Baseline & $4^{\text {th }}$ week & $8^{\text {th }}$ week & $\begin{array}{l}\text { Statistic } \\
\text { F-ratio } \dagger\end{array}$ & $p$ value \\
\hline Parametric & $\bar{x} \pm S D$ & $\bar{x} \pm S D$ & $\bar{x} \pm S D$ & $t$ value & \\
\hline \multicolumn{6}{|l|}{ Pain intensity } \\
\hline Right now & $4.1 \pm 1.8$ & $2.2 \pm 1.4$ & $0.6 \pm 0.8$ & 107.4 & $0.001^{*}$ \\
\hline Average & $4.8 \pm 1.1$ & $3.0 \pm 1.1$ & $0.9 \pm 0.9$ & 465.6 & $0.001^{*}$ \\
\hline At its best & $2.6 \pm 1.3$ & $1.4 \pm 1.3$ & $0.2 \pm 0.4$ & 68.88 & $0.001^{*}$ \\
\hline At its worst & $6.9 \pm 1.4$ & $4.8 \pm 1.7$ & $2.1 \pm 1.6$ & 425.7 & $0.001^{*}$ \\
\hline QVAS score & $52.9 \pm 11.4$ & $33.3 \pm 9.6$ & $12.1 \pm 10.1$ & 483.1 & $0.001^{*}$ \\
\hline Activity limitation & $6.8 \pm 4.9$ & $3.7 \pm 2.9$ & $1.1 \pm 1.1$ & 54.45 & $0.001^{*}$ \\
\hline Back muscles endurance & $35.3 \pm 22.5$ & $49.1 \pm 24.2$ & $57.2 \pm 28.8$ & 82.20 & $0.001^{*}$ \\
\hline \multicolumn{6}{|l|}{ General health status } \\
\hline \multicolumn{6}{|l|}{ Scale } \\
\hline Physical function & $45.5 \pm 25.3$ & $68.9 \pm 15.7$ & $77.3 \pm 15.0$ & 24.8 & $0.001^{*}$ \\
\hline Role limitation-physical & $82.5 \pm 17.9$ & $77.7 \pm 17.2$ & $79.3 \pm 17.6$ & 00.66 & 0.427 \\
\hline Bodily pain & $73.0 \pm 15.5$ & $81.4 \pm 15.4$ & $92.7 \pm 11.2$ & 31.55 & $0.001^{*}$ \\
\hline Health perception & $52.5 \pm 23.9$ & $74.1 \pm 18.6$ & $85.9 \pm 14.3$ & 43.4 & $0.001^{*}$ \\
\hline Energy/fatigue & $60.0 \pm 21.4$ & $77.3 \pm 17.8$ & $86.4 \pm 18.9$ & 23.1 & $0.001^{*}$ \\
\hline Social functioning & $87.5 \pm 12.8$ & $81.8 \pm 11.4$ & $75.0 \pm 0.0$ & 21.0 & $0.001^{*}$ \\
\hline Role limitation-emotional & $87.2 \pm 17.2$ & $73.0 \pm 15.0$ & $68.2 \pm 10.3$ & 25.2 & $0.001^{*}$ \\
\hline Mental health & $52.5 \pm 13.7$ & $59.7 \pm 10.6$ & $64.2 \pm 10.1$ & 11.0 & $0.003 *$ \\
\hline \multicolumn{6}{|l|}{ Domain } \\
\hline Mental health & $71.8 \pm 5.97$ & $72.9 \pm 6.01$ & $73.5 \pm 4.71$ & 12.003 & $0.001^{*}$ \\
\hline Physical health & $68.7 \pm 6.1$ & $76.4 \pm 6.4$ & $80.4 \pm 5.71$ & 39.1 & $0.001^{*}$ \\
\hline Non-parametric & MR & MR & MR & $\chi 2 \ddagger$ & \\
\hline Participation restriction & 2.9 & 1.8 & 1.3 & 33.7 & $0.001^{*}$ \\
\hline \multicolumn{6}{|l|}{ Fear avoidance belief } \\
\hline At work & 1.9 & 1.6 & 2.4 & 8.72 & $0.013^{*}$ \\
\hline In physical activities & 1.6 & 1.7 & 2.7 & 24.5 & $0.001 *$ \\
\hline Fear of movement & 1.4 & 1.8 & 2.8 & 21.7 & $0.001^{*}$ \\
\hline
\end{tabular}

* significant difference, $p<0.05$, † repeated measures ANOVA, $\ddagger$ Friedman’s ANOVA

BE-VRG - Back Extension-Virtual Reality Game, $\bar{x}$ - mean, SD - standard deviation, QVAS - Quadruple Visual Analogue Scale, MR - mean ranked

gruence of real and perceived movement as a cortical driver of pain is still controversial [55].

VRGs seem to present a cognitive platform for retraining the brain's ability to correctly code for movement, which in turn may show promising results for chronic LBP management [56]. Thomas et al. [49] submits that an increased dosage of VRG training could lead to sustained cognitive and behavioural changes in patients with high pain-related fears. They recommend that longer (i.e. > 12) sessions of graded exposure to VRG may lead to constant improvement attained during the VRG play that would transfer over to everyday life situations. While in the study by Thomas et al. [49] VRG was applied for over 8-12 sessions, the current study used VRG for about 5-10 minutes for 24 sessions. Thus, the larger dosage of VRG in this study may have facilitated reduced pain output and protective fear of movement response among the patients, downscaling the expectancy of harm and perception of danger.

The comparability in findings between the CBMT and BE-VRG at the $4^{\text {th }}$ and $8^{\text {th }}$ week in the present study may help reduce dependency on therapist's skill for positive outcomes that has been associated with the McKenzie method. Moreover, the proper evaluation and appropriate treatment in the McKenzie therapy are premised on specialized training in the McKenzie MDT [12, 13, 57]. However, the BE-VRG used in this study is not an exact replica of the McKenzie extension therapy, as gliding movement was also part 
of the game. At the $8^{\text {th }}$ week of the study, no significant differences were found in the treatment outcome between the two groups except for higher mean change observed in fear of movement and energy/vitality in the BE-VRG compared with CBMT group. Therefore, BE-VRG has a superior effect in reducing Kp; however, it is inferred that it is more energy-demanding on the patient than the clinic-based method. For example, while CBMT requires that a patient work through up to 10 repetitions of movement, patients in the BEVRG, unknown to them, would have accomplished 90-120 repetitions of extension-glide movements.

In sum, the VRG group obtained a similar decline in most outcome measures as the clinic-based McKenzie group, indicating that the virtual reality platform was as successful as the traditional method. Thus, the findings of this study prove that VRG is an effective and comparable platform to implement McKenzie exercise for patients with chronic LBP. Loman and Quinlan [58] posit that new technologies offer a potential means of enhancing patient engagement in usual care or traditional therapy, as the use of computers and gaming equipment in physiotherapy is progressively more relevant in the medical community. In consonance, Thomas et al. [49] submit that VRG could be an exciting future tool for physiotherapy treatment, certainly constituting a part of a multidimensional biopsychosocial framework that may help patients encode multisensory information more accurately. In addition, the use of BE-VRG can bridge the gap in the non-availability of CBMT facilities, especially in remote settings. However, more research is needed to generate sufficient evidence on the efficacy of VRG-delivered exercises in chronic LBP.

It is noteworthy to state that both groups in this study were comparable in their baseline general characteristics, except for static BEME, PR, fear of movement, as well as in some of the SF-12 items. Because it is plausible that the baseline differences could have influenced outcome, an analysis of covariance was computed for fear of movement and vitality/fatigue (two variables that were significantly different between the groups at the end of the study) to ascertain whether the baseline variables were predictors of outcomes. However, the results indicate that the differences that existed in some of the baseline measures were not significant predictors of the study outcomes. Comparability in baseline measures in clinical trials is believed to lessen the probability that co-founders other than the main intervention would influence outcomes, as baseline characteristics are believed to be predictors of response to treatment in clinical trials for LBP [59]. Nonetheless, Friedman et al. [60] argue that for many measurements, baseline data may not be a true reflection of the participants' condition, as investigators perform baseline assessment usually close to the time of intervention. It is therefore important to stress that though the usage of VRG in rehabilitation makes the process of the intervention more attractive, it is very important to conduct more research to validate the effectiveness of this innovative approach compared with traditional therapies. It is also crucial to note that VRG-aided rehabilitation will not push aside the role of physical therapists in appropriate assessment, prescription, and implementation of exercise.

\section{Conclusions}

Back extension-glide VRG is comparable with the McKenzie extension therapy in its effect on pain and pain-related characteristics, disability, movement impairments, and quality of life. While the BE-VRG has superior effect in reducing Kp, it takes a higher toll on self-reported vitality/energy.

\section{Acknowledgements}

The authors thank Mr. Ogor Anumbor for the technical input in the design of the back extension-glide VRG. This research was partially funded by an African Doctoral Dissertation Research Fellowship re-entry grant awarded by the African Population and Health Research Centre in partnership with the International Development Research Centre (IDRC).

\section{Disclosure statement}

No author has any financial interest or received any financial benefit from this research.

\section{Conflict of interest}

The authors state no conflict of interest.

\section{References}

1. Walker BF, Muller R, Grant WD. Low back pain in Australian adults. Health provider utilization and care seeking. J Manipulative Physiol Ther. 2004;27(5):327335; doi: 10.1016/j.jmpt.2004.04.006.

2. Ehrlich GE. Low back pain. Bull World Health Organ. 2003;81(9):671-676.

3. Demoulin C, Vanderthommen M, Duysens C, Crielaard JM. Spinal muscle evaluation using the Sorensen test: a critical appraisal of the literature. Joint Bone Spine. 2006;73(1):43-50; doi: 10.1016/j.jbspin.2004. 08.002.

4. Hoy D, March L, Brooks P, Blyth F, Woolf A, Bain C, et al. The global burden of low back pain: estimates from the Global Burden of Disease 2010 study. Ann Rheum Dis. 2014;73(6):968-974; doi: 10.1136/annrheumdis2013-204428. 
5. United Nations, Department of Economic and Social Affairs, Population Division. World Population Ageing 2015. New York: United Nations; 2015.

6. Andersson GBJ. The epidemiology of spinal disorders. In: Frymoyer JW, Ducker TB (eds.), The adult spine: principles and practice, $2^{\text {nd }} \mathrm{ed}$. Philadelphia: Lippincott-Raven; 1997; 93-141.

7. Bigos S, Bowyer OR, Braen GR, Brown K, Deyo R, Haldeman $S$, et al. Acute low back problems in adults. Clinical Practice Guideline No. 14. Rockville: Agency for Health Care Policy and Research; 1994.

8. Hayden JA, van Tulder MW, Malmivaara AV, Koes BW. Meta-analysis: exercise therapy for nonspecific low back pain. Ann Intern Med. 2005;142(9):765-775; doi: 10.7326/0003-4819-142-9-200505030-00013.

9. Brukner P, Khan K. Clinical sports medicine. Sydney: McGraw-Hill; 1993.

10. Gracey JH, McDonough SM, Baxter GD. Physiotherapy management of low back pain: a survey of current practice in northern Ireland. Spine. 2002;27(4):406411; doi: 10.1097/00007632-200202150-00017.

11. Moffett J, McLean S. The role of physiotherapy in the management of non-specific back pain and neck pain. Rheumatology. 2006;45(4):371-378; doi: 10.1093/ rheumatology/kei242.

12. Clare HA, Adams R, Maher CG. A systematic review of efficacy of McKenzie therapy for spinal pain. Aust J Physiother. 2004;50(4):209-216; doi: 10.1016/S00049514(14)60110-0.

13. Czajka M, Truszczyńska-Baszak A, Kowalczyk M. The effectiveness of McKenzie method in diagnosis and treatment of low back pain - a literature review. Adv Rehab. 2018;1:5-11; doi: 10.5114/areh.2018.76985.

14. Dansky KH, Palmer L, Shea D, Bowles KH. Cost analysis of telehomecare. Telemed J E Health. 2001;7(3): 225-232; doi: 10.1089/153056201316970920.

15. Cooper RA, Fitzgerald SG, Boninger ML, Brienza DM, Shapcott N, Cooper R, et al. Telerehabilitation: expanding access to rehabilitation expertise. Proc IEEE. 2001;89(8):1174-1191; doi: 10.1109/5.940286.

16. Kato PM, Cole SW, Bradlyn AS, Pollock BH. A video game improves behavioral outcomes in adolescents and young adults with cancer: a randomized trial. Pediatrics. 2008;122(2):e305-e317; doi: 10.1542/peds.2007-3134.

17. Chan YH. Randomised controlled trials (RCTs) - sample size: the magic number? Singapore Med J. 2003;44(4): 172-174.

18. Waddell G. Low back pain: a twentieth century health care enigma. Spine. 1996;21(24):2820-2825; doi: 10.1097/00007632-199612150-00002.

19. McKenzie R, May S. The lumbar spine: mechanical diagnosis and therapy. Waikanae: Spinal Publications; 2003.

20. Aina A, May S, Clare H. The centralization phenomenon of spinal symptoms - a systematic review. Man Ther. 2004;9(3):134-143; doi: 10.1016/j.math.2004.03.004.

21. Pocock SJ. Allocation of patients to treatment in clini- cal trials. Biometrics. 1979;35(1):183-197; doi: 10.2307/ 2529944.

22. Albright J, Allman R, Bonfiglio RP, Conill A, Dobkin B, Guccione AA, et al. Philadelphia Panel evidence-based clinical practice guidelines on selected rehabilitation interventions for low back pain. Phys Ther. 2001;81(10): 1641-1674; doi: 10.1093/ptj/81.10.1641.

23. Von Korff M, Deyo RA, Cherkin D, Barlow W. Back pain in primary care: outcomes at 1 year. Spine. 1993;18(7): 855-862; doi: 10.1097/00007632-199306000-00008.

24. Fairbank JC, Couper J, Davies JB, O’Brien JP. The Oswestry low back pain disability questionnaire. Physiotherapy. 1980;66(8):271-273.

25. Vianin M. Psychometric properties and clinical usefulness of the Oswestry Disability Index. J Chiropr Med.2008;7(4):161-163; doi: 10.1016/j.jcm.2008.07.001.

26. Roland M, Fairbank J. The Roland-Morris Disability Questionnaire and the Oswestry Disability Questionnaire. Spine. 2000;25(24):3115-3124; doi: 10.1097/ 00007632-200012150-00006.

27. Costa LO, Maher CG, Latimer J, Ferreira PH, Pozzi GC, Ribeiro RN. Psychometric characteristics of the Brazilian-Portuguese versions of the Functional Rating Index and the Roland Morris Disability Questionnaire. Spine. 2007;32(17):1902-1907; doi: 10.1097/ BRS.0b013e31811eab33.

28. Ware J Jr, Kosinski M, Keller SD. A 12-Item ShortForm Health Survey: construction of scales and preliminary tests of reliability and validity. Med Care. 1996;34(3):220-233; doi: 10.2307/3766749.

29. Jenkinson C, Layte R, Jenkinson D, Lawrence K, Petersen $\mathrm{S}$, Paice $\mathrm{C}$, et al. A shorter form health survey: can the SF-12 replicate results from the SF-36 in longitudinal studies? J Public Health Med. 1997;19(2):179186; doi: 10.1093/oxfordjournals.pubmed.a024606.

30. Hurst NP, Ruta DA, Kind P. Comparison of the MOS short form-12 (SF12) health status questionnaire with the SF36 in patients with rheumatoid arthritis. Br J Rheumatol. 1998;37(8):862-869; doi: 10.1093/rheumatology/37.8.862.

31. Waddell G, Newton M, Henderson I, Somerville D, Main CJ. A Fear-Avoidance Beliefs Questionnaire (FABQ) and the role of fear-avoidance beliefs in chronic low back pain and disability. Pain. 1993;52(2):157-168; doi: 10.1016/0304-3959(93)90127-B.

32. Pool JJM, Hiralal S, Ostelo RW, van der Veer K, Vlaeyen JWS, Bouter LM, et al. The applicability of the Tampa Scale of Kinesiophobia for patients with sub-acute neck pain: a qualitative study. Qual Quant. 2009;43(5):773780; doi: 10.1007/s11135-008-9203-x.

33. Biering-Sørensen F. Physical measurements as risk indicators for low-back trouble over a one-year period. Spine. 1984;9(2):106-119; doi: 10.1097/00007632198403000-00002.

34. Mbada CE, Ayanniyi O, Adedoyin RA. Reference values of static back extensor muscle endurance in healthy Nigerian adults. Med Princ Pract. 2009;18(5):345-350; doi: 10.1159/000226285. 
35. McKenzie R. Treat your own back. Waikanae: Spinal Publications; 1990.

36. Hoy D, Bain C, Williams G, March L, Brooks P, Blyth F, et al. A systematic review of the global prevalence of low back pain. Arthritis Rheum. 2012;64(6):2028-2037; doi: 10.1002/art.34347.

37. Adams MA, May S, Freeman BJ, Morrison HP, Dolan P. Effects of backward bending on lumbar intervertebral discs. Relevance to physical therapy treatments for low back pain. Spine. 2000;25(4):431-437; doi: 10.1097/ 00007632-200002150-00007.

38. Fiebert I, Keller CD. Are “passive” extension exercises really passive? J Orthop Sports Phys Ther. 1994;19(2): 111-116; doi: 10.2519/jospt.1994.19.2.111.

39. Cherkin DC, Deyo RA, Battié M, Street J, Barlow W. A comparison of physical therapy, chiropractic manipulation, and provision of an educational booklet for the treatment of patients with low back pain. N Engl J Med. 1998;339(15):1021-1029; doi: 10.1056/NEJM1998100 83391502 .

40. Machado LA, de Souza MV, Ferreira PH, Ferreira ML. The McKenzie method for low back pain: a systematic review of the literature with a meta-analysis approach. Spine. 2006;31(9):E254-E262; doi: 10.1097/01.brs.0000 214884.18502.93.

41. Mooney V. Training low back pain with exercise. Musculoskel Med. 1995;12:24-34.

42. Moffett JK, Jackson DA, Gardiner ED, Torgerson DJ, Coulton S, Eaton S, et al. Randomized trial of two physiotherapy interventions for primary care neck and back pain patients: 'McKenzie' vs brief physiotherapy pain management. Rheumatology. 2006;45(12):15141521; doi: 10.1093/rheumatology/kel339.

43. Ordway RN, Seymour RJ, Donelson RG, Hojnowski LS, Edwards WT. Cervical flexion, extension, protrusion, and retraction. A radiographic segmental analysis. Spine. 1999;24(3):240-247; doi: 10.1097/00007632-199902 010-00008.

44. Quinnel RC, Stockdale HR, Willis DS. Observations of pressures within normal discs in the lumbar spine. Spine. 1983;8(2):166-169; doi: 10.1097/00007632198303000-00007.

45. Kopp JR, Alexander AH, Turocy RH, Levrini MG, Lichtman DM. The use of lumbar extension in the evaluation and treatment of patients with acute herniated nucleus pulposus. A preliminary report. Clin Orthop RelatRes. 1986;202:211-218; doi: 10.1097/00003086198601000-00028.

46. Magnusson SP, Simonsen EB, Aagaard P, Sørensen H, Kjaer M. A mechanism of altered flexibility in human skeletal muscle. J Physiol. 1996;497(Pt 1):291-298; doi: 10.1113/jphysiol.1996.sp021768.

47. Mbada C, Olaoye M, Ayanniyi O, Johnson O, Odole A, Dada O. Comparative efficacy of clinic-based and telerehabilitation application of Mckenzie therapy in lowback pain. Arch Phys Med Rehabil. 2017;98(10):e46e47; doi: 10.1016/j.apmr.2017.08.143.
48. Wand BM, Tulloch VM, George PJ, Smith AJ, Goucke R, O’Connell NE, et al. Seeing it helps: movement-related back pain is reduced by visualization of the back during movement. Clin J Pain. 2012;28(7):602-608; doi: 10.1097/AJP.0b013e31823d480c.

49. Thomas JS, France CR, Applegate ME, Leitkam ST, Walkowski S. Feasibility and safety of a virtual reality dodgeball intervention for chronic low back pain: a randomized clinical trial. J Pain. 2016;17(2):1302-1317; doi: 10.1016/j.jpain.2016.08.011.

50. Flöel A. tDCS-enhanced motor and cognitive function in neurological diseases. Neuroimage. 2014;85(Pt 3): 934-947; doi: 10.1016/j.neuroimage.2013.05.098.

51. López-de-Uralde-Villanueva I, Muñoz-García D, GilMartínez A, Pardo-Montero J, Muñoz-Plata R, AnguloDíaz-Parreño $S$, et al. A systematic review and metaanalysis on the effectiveness of graded activity and graded exposure for chronic nonspecific low back pain. Pain Med. 2016;17(1):172-188; doi: 10.1111/pme.12882.

52. Rainville J, Hartigan C, Martinez E, Limke J, Jouve C, Finno M. Exercise as a treatment for chronic low back pain. Spine J. 2004;4(1):106-115; doi: 10.1016/S1529 -9430(03)00174-8.

53. Burke D, Bartley K, Woodforth IJ, Yakoubi A, Stephen JP. The effects of a volatile anaesthetic on the excitability of human corticospinal axons. Brain. 2000; 123(Pt 5):992-1000; doi: 10.1093/brain/123.5.992.

54. Bowering KJ, O’Connell NE, Tabor A, Catley MJ, Leake HB, Moseley GL, et al. The effects of graded motor imagery and its components on chronic pain: a systematic review and meta-analysis. J Pain. 2013;14(1):3-13; doi: 10.1016/j.jpain.2012.09.007.

55. Saposnik G, Levin M, Outcome Research Canada (SORCan) Working Group. Virtual reality in stroke rehabilitation: a meta-analysis and implications for clinicians. Stroke. 2011;42(5):1380-1386; doi: 10.1161/ STROKEAHA.110.605451.

56. Wand BM, O’Connell NE, Di Pietro F, Bulsara M. Managing chronic nonspecific low back pain with a sensorimotor retraining approach: exploratory multiple-baseline study of 3 participants. Phys Ther. 2011; 91(4):535-546; doi: 10.2522/ptj.20100150.

57. Miller J, Herbowy S. The McKenzie approach. Rehab Manag. 2002;15(7):40-44.

58. Loman NJ, Quinlan AR. Poretools: a toolkit for analyzing nanopore sequence data. Bioinformatics. 2014; 30(23):3399-3401; doi: 10.1093/bioinformatics/btu555.

59. Underwood MR, Morton V, Farrin A, UK BEAM Trial Team. Do baseline characteristics predict response to treatment for low back pain? Secondary analysis of the UK BEAM dataset. Rheumatology. 2007;46(8): 1297-1302; doi: 10.1093/rheumatology/kem113.

60. Friedman LM, Furberg CD, DeMets DL. Baseline assessment. In: Friedman LM, Furberg CD, DeMets DL (eds.), Fundamentals of clinical trials. New York: Springer; 2010; 169-182. 